Buckley Rachel (Orcid ID: 0000-0002-5356-5537)

Jacobs Heidi (Orcid ID: 0000-0001-7620-3822)

\title{
Sex mediates relationships between regional tau pathology and cognitive decline
}

Running head: Sex, regional tau pathology and cognitive decline

Authors: Rachel F Buckley*1,2,3, PhD, Matthew R Scott*1, BA, Heidi IL Jacobs ${ }^{4,5}$, PhD, Aaron P

Schultz $^{1}$, PhD, Michael J Properzi ${ }^{1}$, BEng, Rebecca E Amarglio ${ }^{1,2}$, PhD, Timothy J Hohman ${ }^{6}$, PhD, Danielle V Mayblyum ${ }^{5}$, BS, Zoe B Rubinstein ${ }^{5}$, BA, Lyssa Manning ${ }^{1}$, MA, Bernard J Hanseeuw ${ }^{1,7}$, MD PhD, Elizabeth C Mormino ${ }^{8}$, PhD, Dorene M Rentz ${ }^{1,2}$, PhD, Keith A Johnson ${ }^{2,5}$, MD, Reisa A Sperling ${ }^{1,2}$, $\mathrm{MD}^{\# \# \#}$

*Both authors contributed equally to this work

${ }^{1}$ Department of Neurology, Massachusetts General Hospital and Harvard Medical School, Boston, MA, USA ${ }^{2}$ Center for Alzheimer Research and Treatment, Department of Neurology, Brigham and Women's Hospital, Boston, Massachusetts, USA

${ }^{3}$ Melbourne School of Psychological Science, University of Melbourne, Victoria, Australia

${ }^{4}$ Faculty of Health, Medicine and Life Sciences, School for Mental Health and Neuroscience, Alzheimer Centre Limburg, Maastricht University, Maastricht, The Netherlands

${ }^{5}$ Division of Nuclear Medicine and Molecular Imaging, Department of Radiology, Massachusetts General Hospital/Harvard Medical School, Boston, MA

${ }^{6}$ Vanderbilt Memory \& Alzheimer’s Center, Department of Neurology, Vanderbilt University Medical Center, Nashville, TN, USA

${ }^{7}$ Department of Neurology, Cliniques Universitaires Saint-Luc, Institute of Neuroscience, Université

Catholique de Louvain, 10 Avenue Hippocrate, 1200 Brussels, Belgium

${ }^{8}$ Department of Neurology, Stanford University, CA, USA

\section{Corresponding Author:}

Reisa Sperling

Harvard Aging Brain Study,

Department of Neurology, Massachusetts General Hospital

149 13th Street, Charlestown, MA, USA, 02129

reisa@bwh.harvard.edu

Phone: 617-525-8675

\#A complete listing of the Harvard Aging Brain Study investigators can be found at: http://nmr.mgh.harvard.edu/lab/harvardagingbrain/aboutus

\#"Some data used in preparation of this article were obtained from the Alzheimer's Disease Neuroimaging Initiative (ADNI) database (adni.loni.usc.edu). As such, the investigators within the ADNI contributed to the design and implementation of ADNI and/or provided data but did not participate in analysis or writing of this report. A complete listing of ADNI can be found at: http://adni.loni.usc.edu/wp-content/uploads/how_to_apply/ADNI_Acknowledgement_List.pdf

This is the author manuscript accepted for publication and has undergone full peer review but has not been through the copyediting, typesetting, pagination and proofreading process, which may lead to differences between this version and the Version of Record. Please cite this article as doi: $10.1002 /$ ana. 25878 
Characters in title \& running head: 78 characters \& 43 characters

Abstract word count: 248 words

Introduction: 389 words

Discussion: 1390 words

Full manuscript word count: 3607 words

References: 45

Tables: 1 Figures: 7

\begin{abstract}
Objective: The goal of this study was to examine sex differences in tau distribution across the brain of older adults, using positron emission tomography (PET), and investigate how these differences might associate with cognitive trajectories.

Methods: Participants were 343 clinically-normal individuals (Female,58\%; 73.8[8.5] years) and 55 individuals with MCI (Female,38\%; 76.9[7.3] years) from the Harvard Aging Brain Study and the Alzheimer's Disease Neuroimaging Initiative. We examined ${ }^{18}$ F-Flortaucipir (FTP)-PET signal across 41 cortical and subcortical regions of interest (ROIs). Linear regression models estimated the effect of sex on FTP-signal for each ROI after adjusting for age and cohort. We also examined interactions between sex*A $\beta$-PET(+/-) and sex*APOE 4 status. Linear mixed models estimated the moderating effect of sex on the relationship between a composite of sex-differentiated tau ROIs and cognitive decline.

Results: Females showed significantly higher FTP-signal than males across multiple regions of the cortical mantle $(\mathrm{p}<0.007)$. $A \beta$-moderated sex differences in tau signal were localized to medial and inferio-lateral temporal regions $(\mathrm{p}<0.007)$; $\mathrm{A} \beta+$ females exhibited greater FTP-signal than other groups. APOE $\varepsilon 4$-moderated sex differences in FTP-signal were only found in the lateral occipital lobe. Females with higher FTP-signal in composite ROI exhibited faster cognitive decline than males $(\mathrm{p}=0.04)$.

Interpretation: Tau vulnerability in females is not just limited to the medial temporal lobe and significantly contributed to greater risk of faster cognitive decline. Interactive effects of sex and A $\beta$ were predominantly localized in the temporal lobe, however, sex differences in extra-temporal tau highlights the possibility of accelerated tau proliferation in females with the onset of clinical symptomatology.
\end{abstract}




\section{Introduction}

Growing evidence suggests females exhibit higher levels of tau burden than males ${ }^{1-7}$; this is particularly apparent in those with genetic risk for sporadic Alzheimer's disease (e.g., apolipoprotein $\varepsilon 4$ [APOE $\varepsilon 4$ ] carriers $)^{3}$ or with elevated levels of $\beta$-amyloid (A $\beta$ ) burden ${ }^{5}$. Levels of both CSF total tau and p-tau are higher in female APOE $\varepsilon 4$ carriers across the cognitive impairment spectrum ${ }^{1}$, including those who are clinically normal ${ }^{3}$. Post-mortem findings also observe a greater amount of tau tangles in females than males $^{6,7}$, as supported by a recent meta-analysis of post-mortem samples across multiple cohorts ${ }^{3}$. While in vivo CSF findings are consistent, this modality does not provide regionally specific information about tau burden.

Relatively few tau-PET findings reports have focused on topographical sex differences in tau signal ${ }^{8}$. We recently found that clinically-normal older females with higher levels of global A $\beta$ exhibit higher levels of entorhinal tau burden than males ${ }^{5}$. Our findings, however, were limited to a priori regions of interest (ROIs), leaving open the question as to whether there existed a broader sex dimorphic topographical distribution of tau-PET across the brain. Other studies have reported sex differences in regional tau-PET, but only within the context of glucose metabolism ${ }^{9}$ or APOE $\varepsilon 4$ status $^{10}$. Taken together, the literature is sparse and has not addressed the question of sex differences in the spatial distribution of tau across the brain. Further, it remains unclear how sex dimorphic tau-PET signal might impact rates of cognitive decline. Understanding these differences, particularly within the context of A $\beta$ levels and APOE $\varepsilon 4$ genetic risk, has implications for pinpointing underlying biological mechanisms driving susceptibility to tau, and the extent to which they are reflecting an Alzheimer's disease (AD)-related pathway or other (e.g., age, inflammation, cardiovascular, hormones, methodology).

The aim of this study was to examine sex differences in tau-PET signal across the brain, both as a main effect and interaction with global A $\beta$ and APOE $\varepsilon 4$ carriage. We hypothesized that females would have higher levels of tau across multiple regions, beyond the entorhinal cortex alone which has been reported in multiple cohorts ${ }^{5,9}$. We hypothesized additional regions of the temporal lobe would be implicated, particularly as they represent the early stages of disease in clinically-normal individuals and those diagnosed with mild cognitive impairment (MCI). We also examined whether a composite of these regions that were sex dimorphic would be associated with sex-moderated rates of cognitive decline.

\section{Methods}

\section{Participants}

Data from 343 participants (199 Female, 58\%; 74[8.5] years, range: 50-94yrs) and 55 individuals with MCI (21 Female, 38\%; 77[7.3] years, range: 60-92yrs) were obtained from the Harvard Aging Brain Study (HABS) and Alzheimer's Disease Neuroimaging Initiative (ADNI; adni.loni.usc.edu accessed in February 2018). Sex was categorized on the basis of self-report in both studies. Initial inclusion criteria for recruitment for both HABS and ADNI have been published previously ${ }^{11,12}$. In the current study, 
participants from both studies were diagnosed as clinically-normal or MCI at the time of their first ${ }^{18} \mathrm{~F}$ Flortaucipir (FTP)-PET scan. A total of 251 individuals were included from HABS and 147 individuals from ADNI. For HABS, the time between the first tau-PET scan and the closest A $\beta$-PET scan was a median (interquartile range) of 43 (7-112) days ( $\max =3=y r s)$. For ADNI, the interval was 7 (2-32) days (max=4.8yrs). 21 participants had a lag of longer than one year between scans (HABS: $n=6$; ADNI: $\mathrm{n}=15$ ). We conducted the procedures for this study under the ethical guidelines stipulated by the Partners Human Research Committee, which is the Institutional Review Board for the Massachusetts General Hospital and Brigham and Women's Hospital. Written consent from all individuals was obtained in each cohort.

\section{APOE Genotyping}

A blood sample was collected in each study for direct genotyping of APOE (heterozygotes and homozygotes for the $\varepsilon 4$ allele were collapsed into one category, with $\varepsilon 2-\varepsilon 4$ individuals removed).

\section{Magnetic Resonance Imaging}

Structural T1-weighted anatomical images closest to each participant's tau-PET scan were utilized in both cohorts. T1-weighted images were then processed with FreeSurfer version 6.0 to identify gray-white as well as pial surfaces and to produce automatic Desikan-Killany cortical and aseg subcortical ROI parcellations $^{13}$, with quality control measures previously described ${ }^{11}$.

\section{A $\beta$ Positron Emission Tomography}

HABS used ${ }^{11} \mathrm{C}$ Pittsburgh compound-B (PiB), while ADNI used ${ }^{18} \mathrm{~F}$-Florbetapir (FBP). The PET acquisition parameters for each study have been published previously ${ }^{14-16}$. Distribution volume ratios (DVRs) were computed using Logan plotting 40 to 60 minutes post injection, and summary measures were computed from a weighted average within a large aggregate cortical ROI consisting of precuneus, rostral anterior cingulate, medial orbitofrontal, superior frontal, rostral middle frontal, inferior parietal, inferior temporal, and middle temporal (which is termed FLR) regions, and referenced to cerebellum grey ${ }^{17}$. In ADNI, FBP cortical summary standardized uptake value ratios (SUVrs) were downloaded from data previously processed by the University of California Berkeley from the LONI data access point (http://adni.loni.usc.edu/). Briefly, FBP SUVrs were calculated by combining retention values across cortical ROIs from lateral and medial frontal, anterior, and posterior cingulate, lateral parietal, and lateral temporal regions, and was referenced to the whole cerebellum ${ }^{14}$. As each cohort used different tracers and processing pipelines, A $\beta$-PET signal was expressed dichotomously using a $1.11 \mathrm{SUVr}$ threshold in $\mathrm{ADNI}^{18}$ and a 1.185 DVR threshold in $\mathrm{HABS}^{19}$, although $\mathrm{A} \beta$-focused analyses were also conducted with the continuous measure within each cohort for validation.

\section{Tau Positron Emission Tomography}


Both studies use the FTP tracer (formerly AV1451 or T807). FTP-PET acquisition parameters for each study have been described elsewhere ${ }^{20}$ (for ADNI see, http://adni.loni.usc.edu/methods/petanalysismethod/pet-analysis/). Processing of FTP-PET imaging data for both HABS and ADNI was completed inhouse processing pipelines ${ }^{20}$. SUVrs were created by referencing to cerebellar grey ${ }^{20,21}$. All FTP ROI data are partial volume corrected using the Geometric Transfer Matrix method (GTM) ${ }^{22}$, although associations involving non-PVC data were also explored. Due to off-target binding with FTP-PET ${ }^{23}$, the choroid plexus, putamen, vermis and brainstem were excluded from analyses, and hippocampal which resulted in 41 ROIs for analysis. Hippocampal FTP signal was residualized from choroid plexus signal. In addition, we explored vertex wise maps, normalized in FreeSurfer subject space with PVC using the MullerGartner Extended method ${ }^{24}$.

\section{Tau Analysis}

Analyses were run in R v3.3.3 (The R Foundation) and MATLAB vR2018b (MathWorks, Inc., Natick, MA). Our primary investigation included a series of linear regression models to examine the main effect of sex, and interactions of interest, on FTP SUVr across 41 cortical and sub-cortical ROIs. For sake of parsimony, and due to a lack of a priori assumptions on lateralization, all ROIs were expressed as bihemispheric. All models covaried for age and cohort. To further investigate the spatial distribution of sex effects, we examined surfaced-based vertex wise maps (full sample, and in A $\beta+$ or APOE 44 carriers). As with our previous study ${ }^{5}$, we did not include a three-way sex*A $\beta^{*} A P O E \varepsilon 4$ interactions due to low statistical power. The models were:

Model 1: FTP Sex + Age + Cohort*

Model 2A: FTP $\sim$ Sex + A $\beta$ Group + Age + Cohort* [main effects only]

Model 2B: FTP $\sim$ Sex $X$ A $\beta$ Group + Age + Cohort* [fully factorial]

Model 3: FTP Sex X APOE $\varepsilon 4+$ Age + Cohort*

*A dichotomous indicator for either HABS or ADNI.

Multiple comparison correction

Principal component analysis was used to determine the parameter for multiple comparison correction. We observed 7 eigenvalues $\geq 1$, thus a correction $\alpha<0.007$ was used for multiple comparisons. False Discovery Rate (FDR) correction was used for vertex-wise maps.

Effects on cognitive decline

To examine the effect of elevated FTP-PET signal on cognitive decline, we created a composite of the tau ROIs that differentiated significantly between males and females. We also examined a composite of regions that highlighted sex*A $\beta$ regions, as well as a composite that contained regional signal with no sex differences for reference. We then examined the moderating effect of sex on the relationship between these tau composites and cognitive decline on the Preclinical Alzheimer's Cognitive Composite 
(PACC $^{25}$. We included individuals with at least two neuropsychological assessments (n=388). As FTPPET was introduced mid-way through both studies, we examined time relative to PET scan (both retrospective [ $\left.\mathrm{n}_{\text {observations }}=1626\right]$ and prospective $\left[\mathrm{n}_{\text {observations }}=965\right]$ ), with first assessment occurring approximately 3.5 years ( $\mathrm{SD}=2.2$ years) prior to the tau scan and final assessment approximately 2.3 years $(\mathrm{SD}=1.7)$ years after. We covaried for age, diagnosis at time of tau scan, years of education, and cohort, with random intercepts and slopes. We examined PACC change from baseline and included a quadratic time term.

\section{Sensitivity analyses}

We examined the effect of removing individuals with A $\beta$-PET scans greater than one year from their FTP-PET scan. In addition, we examined findings in only clinically-normal individuals, when using nonPVC FTP-PET, and also when covarying for regional bihemispheric cortical thickness or brain volume within each ROI. We also examined interactions between sex*age. Finally, we separately explored main effects of sex within each cohort, covarying for age, to understand the recapitulation of our findings across samples.

\section{Results}

\section{Demographics}

Participant characteristics within each cohort are presented in Table 1 (Supplementary Table A displays diagnostic demographic comparisons). Within HABS, female participants were younger than males. For ADNI, females exhibited slightly higher continuous A $\beta$-PET SUVr than males. Comparison between cohorts revealed that HABS females were marginally younger, had higher MMSE scores and a lower proportion of $A \beta+$ individuals relative to ADNI females. There were no significant follow-up differences for neuropsychological assessments between men $(t=6.01(\mathrm{SE}=2.5)$ years $)$ and women $(t=5.38(2.4)$ years).

\section{---Table 1---}

\section{Main effect of sex}

Females showed significantly higher FTP SUVr signal in the following ROIs (listed by decreasing magnitude of effect): inferior parietal, rostral middle frontal, fusiform, supramarginal, superior parietal, lateral occipital, middle temporal, superior temporal, temporal pole, banks of the superior temporal sulcus, frontal pole, pars orbitalis, caudal middle frontal, pars orbitalis, cuneus, inferior temporal, postcentral, lingual, and entorhinal gyrus (ps <.007; Fig 1, with model $\mathrm{R}^{2}$ included). Estimated SUVr differences between males and females can be found in Supplementary Table B (and for subsequent models). Adjusting for dichotomous A $\beta$-PET attenuated the sex effect in the pars orbitalis, caudal middle frontal, postcentral, lingual, inferior temporal, and entorhinal gyrus to non-significant (ps $<.05$ ). As a post-hoc analysis, we examined a sex*age interaction but found no effect on any ROIs. 
Sex and A interaction on FTP signal

There was no difference in $\mathrm{A} \beta+/$ - status between males and females $(\mathrm{t}(392)=1.66, \mathrm{p}=0.10)$. $\mathrm{A} \beta+$ females however, exhibited higher FTP-signal in the inferior temporal, fusiform, middle temporal, parahippocampal, and isthmus cingulate regions than any other group (Fig 2). Density maps showing the distributions of tau signal across males and females in $A \beta+/$ - groups are shown in Figure 3 . These regions were recapitulated within each cohort when examining continuous $A \beta$.

\section{---Figure 2---}

Sex and APOEE4 interaction on FTP signal

Female APOE\&4 carriers exhibited higher FTP SUVr in the lateral occipital lobe than any other group (Fig 4).

---Figure 3---

\section{Effects on cognitive decline}

Using a composite of the sex differentiated ROIs (gathered from all significant Model 1 outputs), females with higher FTP signal exhibited faster cognitive decline than males $\left(\mathrm{t}_{\text {sex }}{ }^{*}\right.$ tau composite*time $=-2.49, \mathrm{p}=0.01$; Fig 5 and Supplementary Table $\mathrm{C}$ for model estimates), after adjusting for covariates including diagnostic status. We also found a similar effect when examining a composite of the significant regions from the sex*A $\beta$ interaction (gathered from all significant Model 2B outputs; $t_{\text {sex }}{ }^{*}$ tau composite*time $=-2.34, p=0.02$ ). We found no sex*A $\beta_{\text {status }} *$ tau interaction on cognitive decline $(t=-0.92, p=0.36)$. When examining a composite of tau regions that did not show sex differences (anterior and posterior cingulate and precuneus), we did not find an effect of sex*tau on cognitive decline.

\section{---Figure 4---}

\section{Sex differences in tau ROI signal in clinically-normal individuals}

Findings were largely recapitulated in clinically-normal individuals (see Fig 6), and adjusting for A $\beta$-PET exerted little influence. For the sex*A $\beta$ status interaction, a significant effect was observed in the fusiform, inferior temporal, entorhinal, accumbens area and the amygdala; clinically-normal $A \beta+$ females exhibited higher FTP signal than any other group. Clinically-normal female APOE 4 carriers exhibited higher FTP SUVr in the lateral occipital lobe. Examining the effects on cognitive decline in this group $(n=335)$, we found only trend-level effects in the sex-differentiated composite, $t_{\text {sex }} *$ tau composite*time $=-1.64$,

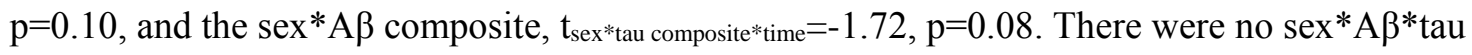
interactions on cognitive decline, which may have reflected low statistical power for detection. 


\section{Sensitivity Analyses}

Excluding individuals with A $\beta$-PET scans greater than one year in duration from the FTP-scan did not alter findings. Using non-PVC FTP signal, significant main effects of sex were observed in similar regions than with PVC, albeit with slightly stronger effects in some cases. When covarying regional cortical thickness or volume in the non-PVC analyses, the only ROI to show attenuated effects was the entorhinal cortex, which maintained a p 0.007. Similarly, covarying for diagnosis or PACC had little influence on the main effect of sex. We examined sex differences in off-target FTP-binding regions such as the skull; for this ROI, females had greater signal than males $(\beta=0.83(0.1), p<0.001)$. Covarying for skull FTP signal (whether PVC or non-PVC), however, did not markedly change the pattern of findings, and in some instances, magnified the sex effect. No significant (corrected or uncorrected) sex*age effect was observed across all FTP-PET PVC ROIs. Moreover, the only sex*age effect in non-PVC FTP signal was limited to the precentral gyrus (uncorrected). Finally, we found that examining each cohort individually largely recapitulated the main effects of sex that were reported when combining both cohorts (Fig 7).

\section{Discussion}

Examining broad sex-related tau vulnerability revealed that females exhibited higher FTP SUVr than males in many temporal and extra-temporal regions. These comprised the parietal, middle frontal, lateral occipital, fusiform, supramarginal, cuneus, banks of the STS, and frontal/temporal pole regions. Many of these regions remained significantly different between the sexes even after adjusting for $A \beta$ status. Amyloid-moderated sex differences in tau signal (sex*A $\beta$ interaction) were largely restricted to the temporal lobe. $A \beta+$ females showed higher FTP signal than $A \beta+$ males and $A \beta$ - individuals in regions such as, the inferior and middle temporal gyrus, the fusiform gyrus, and parahippocampus. Some regions exhibited both main sex effects and amyloid-moderated sex effects on tau signal, such as the inferior temporal, middle temporal, and fusiform gyrus. These findings reveal an AD-specific female vulnerability to temporal lobe tauopathy, such that $A \beta+$ females show higher signal than $A \beta+$ males. In clinicallynormal individuals, $A \beta+$ females displayed elevated signal only in temporal regions of the fusiform, inferior temporal and entorhinal cortices. By contrast, female APOE 44 carriers only showed elevated signal in the occipital region relative to male APOE 44 carriers and non-carriers, regardless of clinical status. Our interpretation of these findings is that AD pathology-related exists to explain sex differences in FTP-PET signal, but this is largely confined to the temporal lobe. Our intention of examining APOE\&4moderated sex effects on tau signal arose from previous studies showing that female APOE 44 carriers have higher levels of CSF tau than male carriers ${ }^{3}$. Interestingly, a sex*APOE 4 interaction did not mirror sex*A $\beta$ findings in this study in that female APOE 44 carriers did not show higher tau signal in the temporal lobe. It is possible that there was not enough statistical power to reliably observe a sex*APOE 4 interaction, and/or there may simply be different sources of variance driving these two markers. Finally, a collection of regions (predominantly extra-temporal) appear to be sex divergent but are unexplained by 
$\mathrm{A} \beta$ burden or $\mathrm{AD}$ genetic risk, suggesting other biological mechanisms at play or a potential source of error.

We also found that sex-differentiated cortical patterns of FTP signal translated to faster cognitive decline in females. The interpretation of our cognitive findings are varied: a) that females can 'survive' (without diagnosis) for longer with medial temporal tau deposition, but once tau has spread to the neocortex an accelerated pattern of decline is expected, or b) that men with neocortical tau distribution have already progressed to a dementia diagnosis and are not represented in these models.

Findings in larger ROIs were largely consistent within each sample and were found to exist in analyses examining PVC or non-PVC, whether or not brain atrophy was covaried. Many of the aforementioned regions have been posited as particularly susceptible to higher FTP signal ${ }^{9,25,26}$ and show associations with greater cortical thinning and CSF p-tau levels ${ }^{26}$, supporting the notion that they are proximal to neurodegenerative processes. Previous studies also show higher FTP signal in these regions in those with elevated $\mathrm{A} \beta$ burden $^{27,28}$, supporting the notion of a female vulnerability to tau in AD vulnerable regions. Some regions that showed sex differences (e.g. frontal and temporal pole) are small and attract signal-tonoise issues. We found smaller effect sizes associated with these regions, and unsurprisingly were more affected by partial-volume correction. As such, findings in these regions should be interpreted with caution. Importantly, clinically-normal individuals could recapitulate the overall pattern of findings, suggesting that they were not solely driven by cognitive impairment.

Similar to our previous work $^{5}$, we found that clinically-normal older $A \beta+$ females showed greater tau in medial temporal regions than males, suggesting that $A \beta$-related susceptibility follows the early topographical patterns of the AD pathological cascade ${ }^{29-33}$. Although studies of CSF total tau and p-tau have often reported a sex-APOE effect, we did not find any regions to robustly show this effect, except in the lateral occipital lobe. This was supported by our previous analysis of a priori regions that only showed weak sex*APOE\&4 interactive effects on FTP SUVr ${ }^{5}$. Another rationale for elevated FTP SUVr in females may be due to non-specific binding ${ }^{23}$. Binding in extra-cortical hotspots such as the lateral occipital, middle temporal, and superior and inferior parietal lobes have been reported beyond the common FTP-related culprits (e.g., choroid plexus, skull, meninges) ${ }^{23}$. It remains unclear why females might express additional susceptibility to non-specific binding. Additional sensitivity analyses revealed sex differences in skull FTP signal in both cohorts, although covarying for this signal did not appreciably change our findings. This does raise some interesting questions about whether this difference is arising from off-target binding, or spillover from signal in regions of interest. A comprehensive exploration and assessment of the extent to which other contributors may drive sex differences in FTP signal is critical to interpreting the biological underpinnings of such.

One question that this study cannot answer is the potential biological mechanism that might drive female vulnerability to tau deposition. Changes in estrogen exposure in postmenopausal females are one potential rationale ${ }^{8,34,35}$. Studies have yet to report on the relationship between endogenous or exogenous estrogen levels and regional tau deposition, however, there are some studies linking $A \beta$ with changes in estrogen, 
suggesting this might have a downstream effect on tau vulnerability ${ }^{36,37}$. Observational studies, however, do not show sex differences in A $\beta$-PET signal ${ }^{19,38}$, although it may be possible that $A \beta$ differences may occur earlier, such as during menopause ${ }^{37}$, which may have a downstream effect on tau deposition. A recent pilot clinical-trial showed that women treated with transdermal 17 $\beta$-estradiol exhibited slightly lower levels of A $\beta$-PET signal than an age-matched placebo group (particularly female APOE 44 carriers) $^{39}$. There is also a possibility that tau vulnerability may be a direct result of estrogen depletion post-menopause, and animal models support this hypothesis. ${ }^{34,40}$ Without direct human-model evidence, this remains unclear. Sex differences exist in risk for metabolic syndrome in this age group, and sex dimorphism in inflammatory markers are well-established ${ }^{41,42}$; considering their link with AD dementia risk $^{43,44}$, these factors could play a role in female tau vulnerability. In addition, it remains unclear the impact of age on these models: it is possible that younger females with higher amyloid burden may express a more aggressive progression of pathology. We were cautious about testing such complicated multi-way interactions when statistical power was lacking, however, we believe a natural next step is to interrogate this question, particularly in light of the fact that the menopausal phase begins, on average, at 42 years of age in the general population.

Finally, it is important to address the issue of survival bias and competing risk for mortality that may be driving sex differences. An examination of density plots of tau deposition in temporal regions (Figure 2D) highlights that females showed higher levels of tau across all these regions and not greater numbers of females at higher tau levels. That is, males with higher tau did not exist in either sample. Women were slightly younger than men in the current study, and recent evidence suggests that tau paradoxically increases in younger-old clinically-normal $A \beta+$ individuals ${ }^{45}$. Further, males with higher levels of tau in these regions may not be included for various reasons: (1) stringent study inclusion criteria (i.e. lower cardiovascular risk), (2) faster rates of progression to dementia or death in males with higher temporal tau, or (3) males may be less likely to join observation studies or opt-in for PET scanning.

The strength of this study is a combined analysis of two independent cohorts, which can provide statistical power to examine effects that may appear across multiple ROIs. One limitation, however, is the extent to which sex differences are a representation of PET methodological effects. We examined morphological differences, PVC and non-PVC FTP metrics, however, and did not find evidence of major biases. Another limitation is the lack of generalizability to community populations; individuals from HABS and ADNI have high education, socioeconomic status, and low racial and ethnic diversity. Examining sex differences in regional tau deposition will be critical in more diverse populations.

In conclusion, our findings suggest that females exhibit higher FTP signal than males in multiple brain regions, in earlier stages of disease. Higher FTP signal in these regions translated to accelerated cognitive decline in females relative to males. Examining these findings within the context of longitudinal FTPPET studies could help to address whether males and females with medial temporal tau, or who are $\mathrm{A} \beta+$, have different FTP-PET trajectories across the brain. 


\section{Funding and other acknowledgements}

The authors thank the participants who volunteered their valuable time to these studies. The Harvard Aging Brain Study is funded by the National Institute on Aging (P01AG036694) with additional support from several philanthropic organizations. The Alzheimer's Disease Neuroimaging Initiative is funded by the NIA (U19AG024904), the NIBIB, the Canadian IHR, and several philanthropic organizations. Several co-authors' contributions were also supported by career development awards: RFB is supported by a K99/R00 award from NIA; HIL is supported by a Marie-Sklodowska-Curie Global Fellowship within the European Union's Horizon 2020 Research and Innovation Programme; TJH is supported by a K01 award from NIA.

\section{Author contributions}

Study concept and design was carried out by RFB, MRS, REA, ECM, HILJ, BJH, DMR, KAJ, RAS.

Data acquisition and analysis was carried out by RFB, MRS, MJP, TJH, APS, DVM, ZBR, LM. Drafting the manuscript and figures was conducted RFB, MRS, RAS.

\section{Conflict of interest}

Nothing to report 


\section{References}

1. Altmann A, Tian L, Henderson VW, Greicius MD. Sex modifies the APOE-related risk of developing Alzheimer disease. Annals of neurology 2014;75:563-573.

2. Damoiseaux JS, Seeley WW, Zhou J, et al. Gender Modulates the APOE $\varepsilon 4$ Effect in Healthy Older Adults: Convergent Evidence from Functional Brain Connectivity and Spinal Fluid Tau Levels. The Journal of Neuroscience 2012;32:8254-8262.

3. Hohman T, Dumitrescu L, Barnes L, et al. Sex-specific effects of Apolipoprotein E on cerebrospinal fluid levels of tau. JAMA Neurology 2018;75:989-998.

4. Buckley RF, Mormino EC, Chhatwal J, et al. Associations between baseline amyloid, sex, and APOE on subsequent tau accumulation in cerebrospinal fluid. Neurobiology of Aging 2019;78:178-185.

5. Buckley R, Mormino E, Rabin J, et al. Sex differences in the association between regional tau and global amyloid PET. JAMA Neurol 2019;76:542-551.

6. Barnes LL, Wilson RS, Bienias JL, Schneider JA, Evans DA, Bennett DA. Sex differences in the clinical manifestations of Alzheimer disease pathology. Archives of general psychiatry 2005;62:685-691.

7. Oveisgharan S, Arvanitakis Z, Yu L, Farfel J, Schneider JA, Bennett DA. Sex differences in Alzheimer's disease and common neuropathologies of aging. Acta Neuropathologica 2018.

8. Ferretti MT, Iulita MF, Cavedo E, et al. Sex differences in Alzheimer disease- the gateway to precision medicine. Nature Reviews Neurology 2018;14:457-469.

9. Ramanan VK, Castillo AM, Knopman DS, et al. Association of Apolipoprotein E $\varepsilon 4$, Educational Level, and Sex With Tau Deposition and Tau-Mediated Metabolic Dysfunction in Older Adults. JAMA Network Open 2019;2:e1913909-e1913909.

10. Paranjpe M, Liu M, Paranjpe I, Wang R, Benzinger T, Zhou Y. Sex Modulates the ApoE $\varepsilon 4$ Effect on Tau 18F-AV-1451 PET Imaging in Individuals with Normal Aging and Mild Cognitive Impairment. Journal of Nuclear Medicine 2019;60:253-253.

11. Dagley A, LaPoint M, Huijbers W, et al. Harvard Aging Brain Study: Dataset and accessibility. NeuroImage 2017;144:255-258.

12. Aisen P. Cognitive/clinical endpoints for pre-dementia AD trials. The journal of prevention of Alzheimer's disease 2015;2:82.

13. Mormino EC, Betensky RA, Hedden T, et al. Synergistic effect of $\beta$-amyloid and neurodegeneration on cognitive decline in clinically normal individuals. JAMA Neurology 2014;71:13791385.

14. Landau SM, Mintun MA, Joshi AD, et al. Amyloid deposition, hypometabolism, and longitudinal cognitive decline. Annals of neurology 2012;72:578-586.

15. Landau SM, Lu M, Joshi AD, et al. Comparing positron emission tomography imaging and cerebrospinal fluid measurements of $\beta$-amyloid. Annals of neurology 2013;74:826 -836.

16. Mormino EC, Betensky RA, Hedden T, et al. Amyloid and APOE $\varepsilon 4$ interact to influence shortterm decline in preclinical Alzheimer disease. Neurology 2014;82:1760-1767.

17. Joshi AD, Pontecorvo MJ, Clark CM, et al. Performance characteristics of amyloid PET with florbetapir F 18 in patients with Alzheimer's disease and cognitively normal subjects. Journal of Nuclear Medicine 2012;53:378-384.

18. Buckley RF, Mormino EC, Amariglio RE, et al. Sex, Amyloid, and APOEe4 and risk of cognitive decline in preclinical Alzheimer's disease: findings from three well-characterized cohorts. Alzheimer's \& Dementia 2018;14:1193-1203. 
19. Johnson KA, Schultz A, Betensky RA, et al. Tau positron emission tomographic imaging in aging and early Alzheimer disease. Annals of neurology 2016;79:110-119.

20. Becker JA, Hedden T, Carmasin J, et al. Amyloid- $\beta$ associated cortical thinning in clinically normal elderly. Annals of Neurology 2011;69:1032-1042.

21. Rousset OG, Ma Y, Evans AC. Correction for partial volume effects in PET: principle and validation. The journal of nuclear medicine 1998;39:904.

22. Baker SL, Maass A, Jagust WJ. Considerations and code for partial volume correcting [18F]-AV1451 tau PET data. Data in Brief 2017;15:648-657.

23. Greve DN, Salat DH, Bowen SL, et al. Different partial volume correction methods lead to different conclusions: An 18 F-FDG-PET study of aging. Neuroimage 2016;132:334-343.

24. Donohue MC, Sperling RA, Salmon DP, et al. The preclinical alzheimer cognitive composite: Measuring amyloid-related decline. JAMA Neurology 2014;71:961-970.

25. Schultz SA, Gordon BA, Mishra S, et al. Widespread distribution of tauopathy in preclinical Alzheimer's disease. Neurobiology of Aging 2018;72:177-185.

26. Maass A, Landau S, Baker SL, et al. Comparison of multiple tau-PET measures as biomarkers in aging and Alzheimer's Disease. NeuroImage 2017;157:448-463.

27. Fortea J, Vilaplana E, Alcolea D, et al. Cerebrospinal fluid $\beta$-amyloid and phospho- tau biomarker interactions affecting brain structure in preclinical Alzheimer disease. Annals of neurology 2014;76:223-230.

28. Schöll M, Lockhart SN, Schonhaut DR, et al. PET imaging of tau deposition in the aging human brain. Neuron 2016;89:971-982.

29. Braak H, Braak E. Frequency of Stages of Alzheimer-Related Lesions in Different Age Categories. Neurobiology of Aging 1997;18:351-357.

30. Delacourte A, David J, Sergeant N, et al. The biochemical pathway of neurofibrillary degeneration in aging and Alzheimer's disease. Neurology 1999;52:1158-1158.

31. Braak H, Rub U, Schultz C, Del Tredici K. Vulnerability of cortical neurons to Alzheimer's and Parkinson's diseases. J Alzheimers Dis 2006;9:35-44.

32. Duyckaerts C. Tau pathology in children and young adults: can you still be unconditionally baptist? : Springer, 2011.

33. Braak H, Braak E. Neuropathological stageing of Alzheimer-related changes. Acta Neuropathol 1991;82:239-259.

34. Brinton RD, Yao J, Yin F, Mack WJ, Cadenas E. Perimenopause as a neurological transition state. Nature Reviews Endocrinology 2015;11:393.

35. Snyder HM, Asthana S, Bain L, et al. Sex biology contributions to vulnerability to Alzheimer's disease: A think tank convened by the Women's Alzheimer's Research Initiative. Alzheimer's \& Dementia 2016;12:1186-1196.

36. Schönknecht P, Pantel J, Klinga K, et al. Reduced cerebrospinal fluid estradiol levels are associated with increased $\beta$-amyloid levels in female patients with Alzheimer's disease. Neuroscience Letters 2001;307:122-124.

37. Mosconi L, Berti V, Quinn C, et al. Sex differences in Alzheimer risk. Brain imaging of endocrine vs chronologic aging 2017;89:1382-1390.

38. Jansen WJ, Ossenkoppele R, Knol DL, et al. Prevalence of cerebral amyloid pathology in persons without dementia: A meta-analysis. JAMA 2015;313:1924-1938. 
39. Kantarci K, Lowe VJ, Lesnick TG, et al. Early postmenopausal transdermal 17ß-estradiol therapy and amyloid- $\beta$ deposition. Journal of Alzheimer's Disease 2016;53:547-556.

40. Grimm A, Biliouris EE, Lang UE, Götz J, Mensah-Nyagan AG, Eckert A. Sex hormone-related neurosteroids differentially rescue bioenergetic deficits induced by amyloid- $\beta$ or hyperphosphorylated tau protein. Cellular and Molecular Life Sciences 2016;73:201-215.

41. Zore T, Palafox M, Reue K. Sex differences in obesity, lipid metabolism, and inflammation-A role for the sex chromosomes? Molecular metabolism 2018;15:35-44.

42. Khramtsova EA, Davis LK, Stranger BE. The role of sex in the genomics of human complex traits. Nature Reviews Genetics 2019;20:173-190.

43. Ferreira ST, Clarke JR, Bomfim TR, De Felice FG. Inflammation, defective insulin signaling, and neuronal dysfunction in Alzheimer's disease. Alzheimer's \& dementia 2014;10:S76-S83.

44. Frisardi V, Solfrizzi V, Seripa D, et al. Metabolic-cognitive syndrome: A cross-talk between metabolic syndrome and Alzheimer's disease. Ageing Research Reviews 2010;9:399-417.

45. Jack Jr CR, Wiste HJ, Schwarz CG, et al. Longitudinal tau PET in ageing and Alzheimer's disease. Brain 2018;141:1517-1528. 
Table 1. Demographic comparisons between HABS and ADNI cohorts

Figure 1. Main effects of sex on FTP SUVr after adjusting for age and cohort (A) with ROI-based analyses across all participants (corrected $\mathrm{p}<0.007$ ), (B) showing vertex-wise (False Discovery Rate [FDR] corrected $\mathrm{p}=0.016$ ) and $(\mathrm{C})$ showing beta weights and standard errors for each ROI with corresponding model $\mathrm{R}^{2}$ (bars to the right denoting $\mathrm{F}>\mathrm{M}$ ).

Figure 2. Interactive effects of sex and $A \beta+/$ - on FTP SUVr after adjusting for age and cohort (A) with ROI-based analyses within $A \beta+$ participants only $(n=129$; corrected $p<0.007)$, (B) $\beta$ standardized and SE for each ROI with corresponding model $\mathrm{R}^{2}$ (bars to the right denoting $\mathrm{F}>\mathrm{M}$ ), and (C) model estimates of FTP SUVr in the inferior temporal cortex (with 95\% CI) in males and females with A $\beta$-PET+/-

Figure 3. Density maps depicting the distributions of FTP-signal in regions that are differentiated by a sex*A $\beta$ interaction

Figure 4. Interactive effects of sex and APOE\&4 on FTP SUVr after adjusting for age and cohort (A) with ROI-based analyses within APOE $\varepsilon 4$ carriers only $(n=109$; corrected $\mathrm{p}<0.007)$, (B) $\beta$ standardized and SE for each ROI with corresponding model $\mathrm{R}^{2}$ (bars to the right denoting $\mathrm{F}>\mathrm{M}$ ), and (C) model estimates (with 95\% CI) of FTP SUVr in males and females with and without APOE 4 carriage.

Figure 5. Spaghetti plot of cognitive trajectories over time in clinically-normal individuals $\left(0^{\text {th }}\right.$ time represents the first FTP-PET scan) and faceted by a tertile split of composite FTP-PET signal (top = metaROI, middle = meta temporal ROI, bottom = reference-ROI) with quadratic fit curves stratified by sex

Figure 6. Main effects of sex on FTP SUVr signal in clinically-normal individuals only, showing beta weights and standard errors for each ROI with corresponding model $\mathrm{R}^{2}$ (bars to the right denoting $\mathrm{F}>\mathrm{M}$ ). Red indicates multiple comparison significance $\mathrm{p}<.007$, purple indicates $\mathrm{p}<.05$ and blue indicates subthreshold estimates. 
Figure 7. Main effect of sex on FTP SUVr after adjusting for age and cohort [ROI-based analyses]: (A) ADNI uncorrected (B) ADNI corrected, (C) HABS uncorrected, and (D) HABS corrected 


\section{Please wait...}

If this message is not eventually replaced by the proper contents of the document, your PDF viewer may not be able to display this type of document.

You can upgrade to the latest version of Adobe Reader for Windows®, Mac, or Linux® by visiting http://www.adobe.com/go/reader_download.

For more assistance with Adobe Reader visit http://www.adobe.com/go/acrreader.

Windows is either a registered trademark or a trademark of Microsoft Corporation in the United States and/or other countries. Mac is a trademark
of Apple Inc., registered in the United States and other countries. Linux is the registered trademark of Linus Torvalds in the U.S. and other countries. 


\section{W) WILEY}

\section{COLOR REPRODUCTION IN YOUR ARTICLE}

These proofs have been typeset using the original figure files transmitted to production when this article was accepted for publication. Please review and mark your approval of each figure individually within your proof corrections. Should you need further assistance, please contact by e-mail dhineline@wiley.com

Because of the high cost of color printing we can only print figures in color if authors cover the expense. If you have submitted color figures please indicate your consent to cover the cost on the table listed below by marking the box corresponding to the approved cost on the table. The first color figure is \$650 USD and subsequent color figures are an additional $\$ 400$ USD.

Please note, all color images will be reproduced online at no charge, whether or not you opt for color printing.

You will be invoiced for color charges once the article has been published in print.

Failure to return this form with your article proofs will delay the publication of your article.

JOURNAL ANA

MS. NO. 20-0312

NO. COLOR FIGURES 7

\section{MANUSCRIPT TITLE Sex mediates relationships between regional tau pathology and cognitive decline}

Rachel F Buckley¹,2,3, PhD, Matthew R Scott*1, BA, Heidi IL Jacobs4,5, PhD, Aaron P Schultz1, PhD, Michael J Properzi1, BEng, Rebecca E

Amarglio1,2, PhD, Timothy J Hohman6, PhD, Danielle V Mayblyum5, BA, Zoe B Rubinstein5, BA, Lyssa Manning1, MA, Bernard J

AUTHOR(S) Aanseeuw1,7, MD PhD, Elizabeth C Mormino8, PhD, Dorene M Rentz1,2, PhD, Keith A Johnson2,5, MD, Reisa A Sperling1,2, MD

\begin{tabular}{|c|c|c|c|c|c|}
\hline No. Color Figures & Color Charge & No. Color Figures & Color Charge & No. Color Figures & Color Charge \\
\hline 1 & $\$ 650$ & $\square$ & $\$ 2250$ & $\square$ & $\$ 3850$ \\
\hline 2 & $\$ 1050$ & $\square$ & $\$ 2650$ & 10 & $\$ 4250$ \\
\hline 3 & $\$ 1450$ & 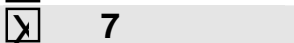 & $\$ 3050$ & 11 & $\$ 4650$ \\
\hline$\square$ & $\$ 1850$ & $\square$ & $\$ 3450$ & 12 & $\$ 5050$ \\
\hline
\end{tabular}

Х Please print my figures color

Please print my figures in black and white

Please print the following figures in color

and convert these figures to black and white

Approved by Reisa Sperling Reisa Sperlye July 22, 2020

Billing Address $\mathrm{MGH}$ at the Navy Yard

E-mail

jmunoz3@bwh.harvard.edu (ASST)

149 13th Street

Telephone

Charlestown, MA 02129

Fax

This article is protected by copyright. All rights reserved. 


\section{Please wait...}

If this message is not eventually replaced by the proper contents of the document, your PDF viewer may not be able to display this type of document.

You can upgrade to the latest version of Adobe Reader for Windows®, Mac, or Linux® by visiting http://www.adobe.com/go/reader_download.

For more assistance with Adobe Reader visit http://www.adobe.com/go/acrreader.

Windows is either a registered trademark or a trademark of Microsoft Corporation in the United States and/or other countries. Mac is a trademark
of Apple Inc., registered in the United States and other countries. Linux is the registered trademark of Linus Torvalds in the U.S. and other countries. 


\section{Please wait...}

If this message is not eventually replaced by the proper contents of the document, your PDF viewer may not be able to display this type of document.

You can upgrade to the latest version of Adobe Reader for Windows®, Mac, or Linux® by visiting http://www.adobe.com/go/reader_download.

For more assistance with Adobe Reader visit http://www.adobe.com/go/acrreader.

Windows is either a registered trademark or a trademark of Microsoft Corporation in the United States and/or other countries. Mac is a trademark
of Apple Inc., registered in the United States and other countries. Linux is the registered trademark of Linus Torvalds in the U.S. and other countries. 


\section{Please wait...}

If this message is not eventually replaced by the proper contents of the document, your PDF viewer may not be able to display this type of document.

You can upgrade to the latest version of Adobe Reader for Windows®, Mac, or Linux® by visiting http://www.adobe.com/go/reader_download.

For more assistance with Adobe Reader visit http://www.adobe.com/go/acrreader.

Windows is either a registered trademark or a trademark of Microsoft Corporation in the United States and/or other countries. Mac is a trademark
of Apple Inc., registered in the United States and other countries. Linux is the registered trademark of Linus Torvalds in the U.S. and other countries. 


\section{Please wait...}

If this message is not eventually replaced by the proper contents of the document, your PDF viewer may not be able to display this type of document.

You can upgrade to the latest version of Adobe Reader for Windows®, Mac, or Linux® by visiting http://www.adobe.com/go/reader_download.

For more assistance with Adobe Reader visit http://www.adobe.com/go/acrreader.

Windows is either a registered trademark or a trademark of Microsoft Corporation in the United States and/or other countries. Mac is a trademark
of Apple Inc., registered in the United States and other countries. Linux is the registered trademark of Linus Torvalds in the U.S. and other countries. 


\section{Please wait...}

If this message is not eventually replaced by the proper contents of the document, your PDF viewer may not be able to display this type of document.

You can upgrade to the latest version of Adobe Reader for Windows®, Mac, or Linux® by visiting http://www.adobe.com/go/reader_download.

For more assistance with Adobe Reader visit http://www.adobe.com/go/acrreader.

Windows is either a registered trademark or a trademark of Microsoft Corporation in the United States and/or other countries. Mac is a trademark
of Apple Inc., registered in the United States and other countries. Linux is the registered trademark of Linus Torvalds in the U.S. and other countries. 


\section{Please wait...}

If this message is not eventually replaced by the proper contents of the document, your PDF viewer may not be able to display this type of document.

You can upgrade to the latest version of Adobe Reader for Windows®, Mac, or Linux® by visiting http://www.adobe.com/go/reader_download.

For more assistance with Adobe Reader visit http://www.adobe.com/go/acrreader.

Windows is either a registered trademark or a trademark of Microsoft Corporation in the United States and/or other countries. Mac is a trademark
of Apple Inc., registered in the United States and other countries. Linux is the registered trademark of Linus Torvalds in the U.S. and other countries. 


\section{Please wait...}

If this message is not eventually replaced by the proper contents of the document, your PDF viewer may not be able to display this type of document.

You can upgrade to the latest version of Adobe Reader for Windows®, Mac, or Linux® by visiting http://www.adobe.com/go/reader_download.

For more assistance with Adobe Reader visit http://www.adobe.com/go/acrreader.

Windows is either a registered trademark or a trademark of Microsoft Corporation in the United States and/or other countries. Mac is a trademark
of Apple Inc., registered in the United States and other countries. Linux is the registered trademark of Linus Torvalds in the U.S. and other countries. 


\section{Please wait...}

If this message is not eventually replaced by the proper contents of the document, your PDF viewer may not be able to display this type of document.

You can upgrade to the latest version of Adobe Reader for Windows®, Mac, or Linux® by visiting http://www.adobe.com/go/reader_download.

For more assistance with Adobe Reader visit http://www.adobe.com/go/acrreader.

Windows is either a registered trademark or a trademark of Microsoft Corporation in the United States and/or other countries. Mac is a trademark
of Apple Inc., registered in the United States and other countries. Linux is the registered trademark of Linus Torvalds in the U.S. and other countries. 


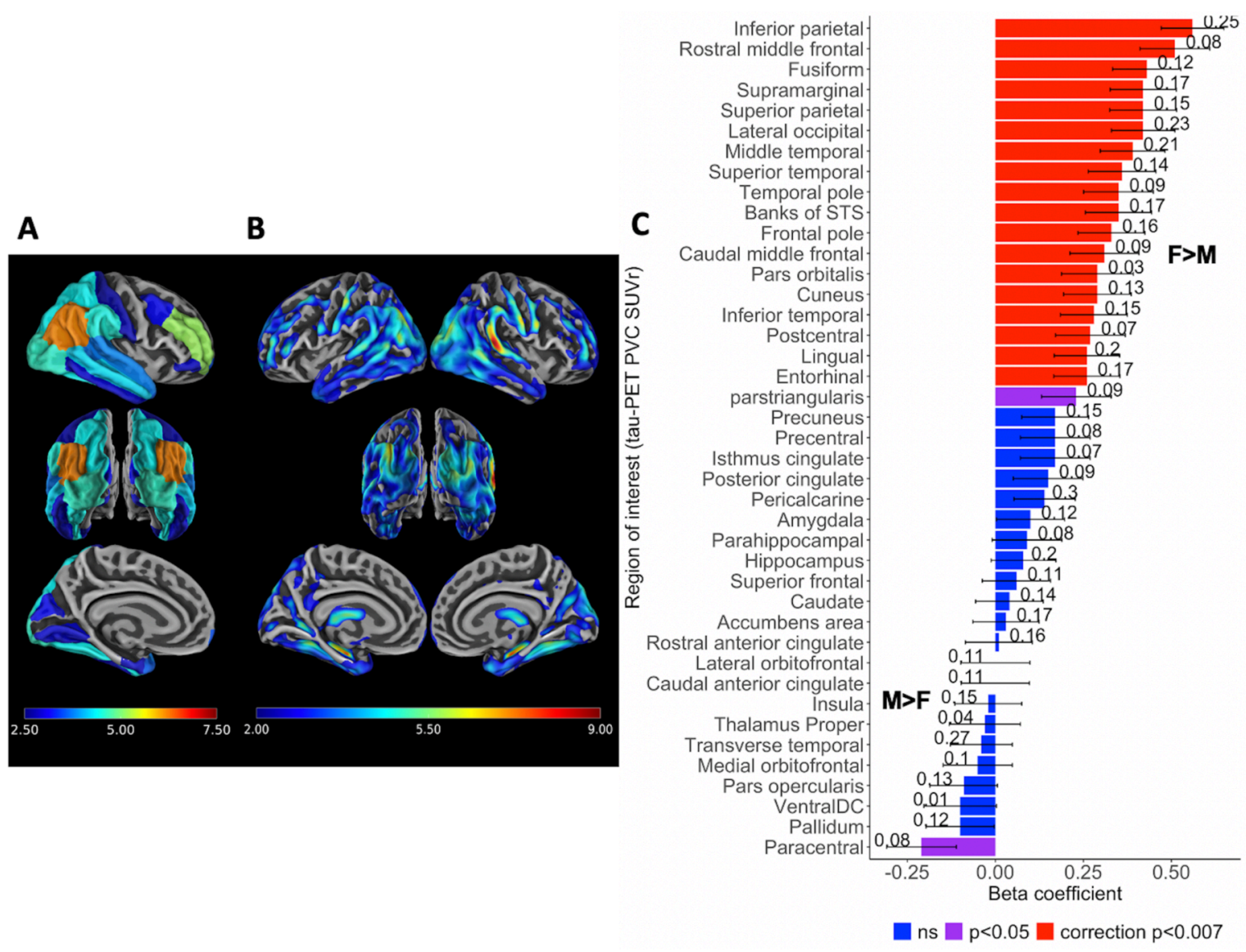

ANA_25878_Fig1_resize.tif

This article is protected by copyright. All rights reserved. 


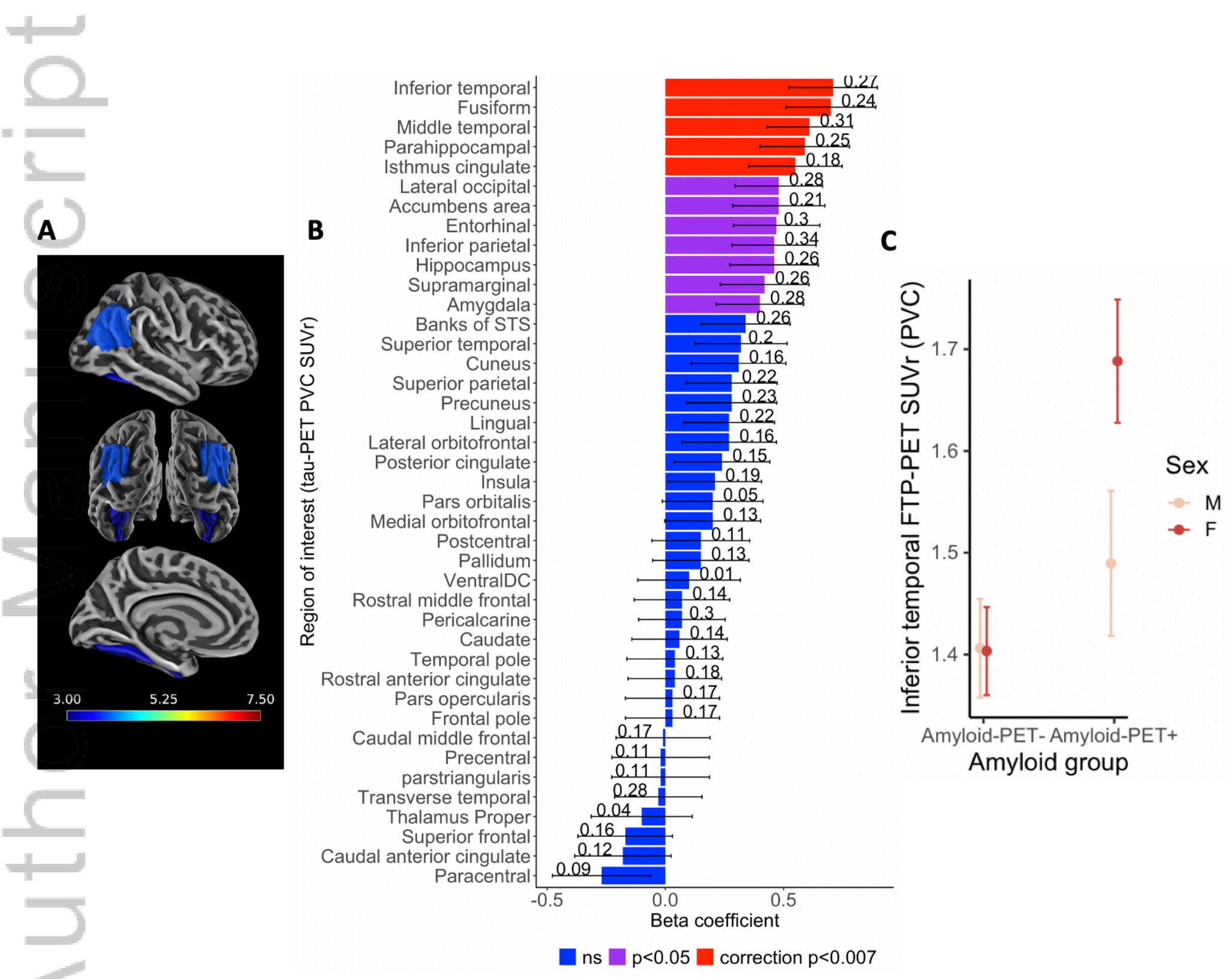

ANA_25878_Fig2_resize.tif 


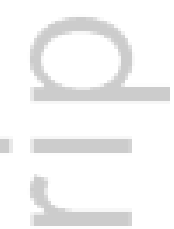

A

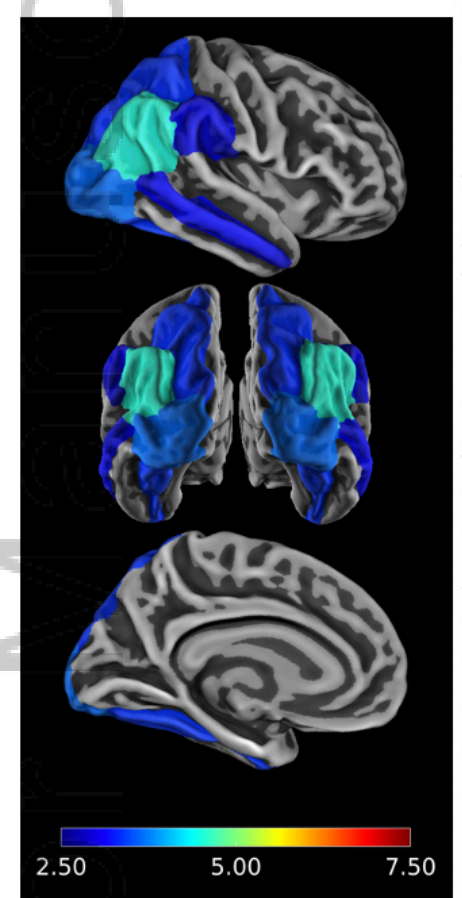

B

D

Lateral occipital Entorhinal Inferior parietal Fusiform Inferior temporal Hippocampus Middle temporal Superior parietal Cuneus Lingual Isthmus cingulate Banks of STS Amygdala

Parahippocampal Thalamus Proper Lateral orbitofrontal

Supramarginal Postcentral

Accumbens area Frontal pole Precuneus Precentral

Temporal pole Rostral middle frontal Pericalcarine Insula Pallidum Superior temporalPars orbitalis Paracentral Medial orbitofrontal Pars opercularis parstriangularis

Posterior cingulate Caudal middle frontal Transverse temporal VentralDC Rostral anterior cingulate Superior frontal Caudate

Caudal anterior cingulate
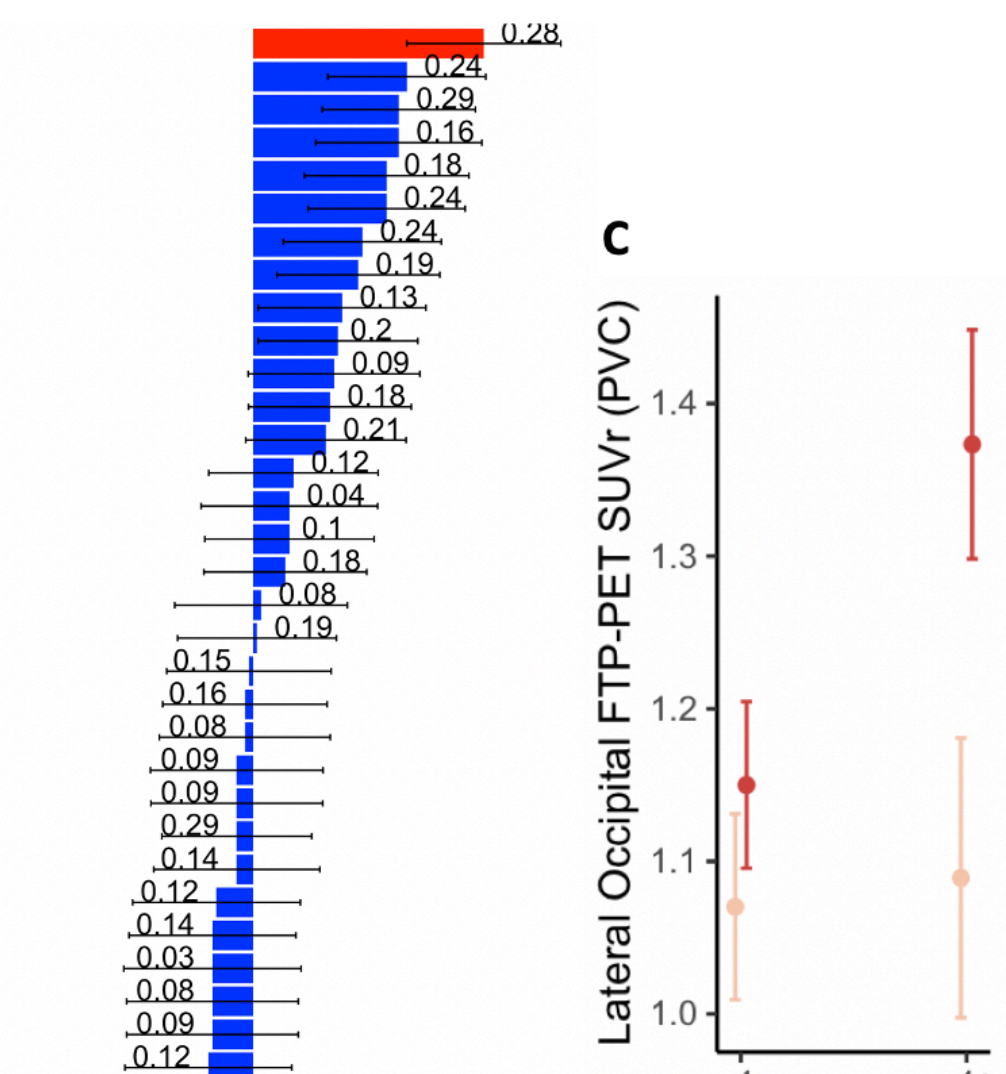

Sex

$\because M$

$\rightarrow F$ 


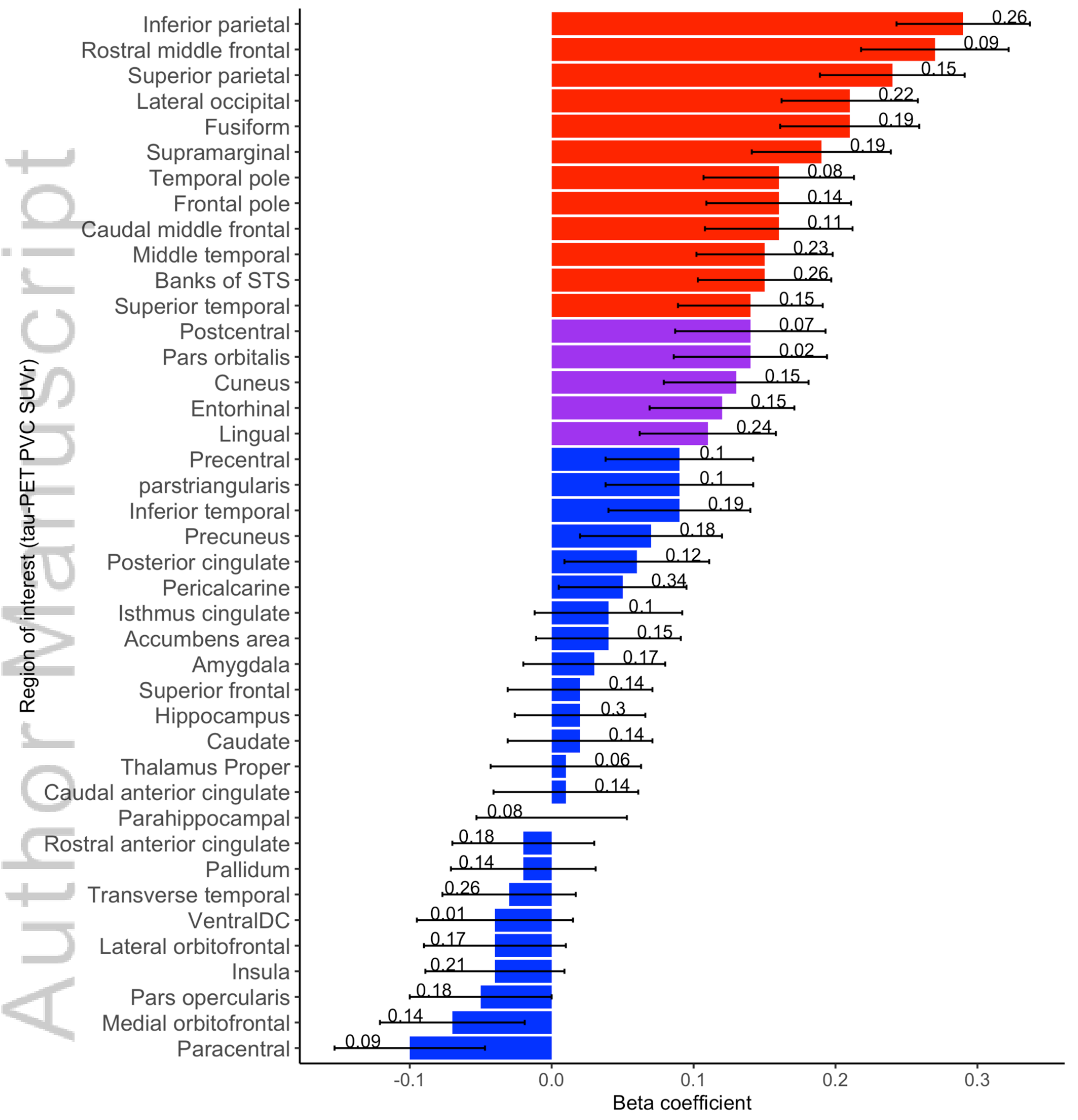

Not significant $p<0.05$ correction $p<0.007$

ANA_25878_Fig6_ANAnew.tiff

This article is protected by copyright. All rights reserved. 


\section{Please wait...}

If this message is not eventually replaced by the proper contents of the document, your PDF viewer may not be able to display this type of document.

You can upgrade to the latest version of Adobe Reader for Windows®, Mac, or Linux® by visiting http://www.adobe.com/go/reader_download.

For more assistance with Adobe Reader visit http://www.adobe.com/go/acrreader.

Windows is either a registered trademark or a trademark of Microsoft Corporation in the United States and/or other countries. Mac is a trademark
of Apple Inc., registered in the United States and other countries. Linux is the registered trademark of Linus Torvalds in the U.S. and other countries. 


\section{Please wait...}

If this message is not eventually replaced by the proper contents of the document, your PDF viewer may not be able to display this type of document.

You can upgrade to the latest version of Adobe Reader for Windows®, Mac, or Linux® by visiting http://www.adobe.com/go/reader_download.

For more assistance with Adobe Reader visit http://www.adobe.com/go/acrreader.

Windows is either a registered trademark or a trademark of Microsoft Corporation in the United States and/or other countries. Mac is a trademark
of Apple Inc., registered in the United States and other countries. Linux is the registered trademark of Linus Torvalds in the U.S. and other countries. 


\section{Please wait...}

If this message is not eventually replaced by the proper contents of the document, your PDF viewer may not be able to display this type of document.

You can upgrade to the latest version of Adobe Reader for Windows®, Mac, or Linux® by visiting http://www.adobe.com/go/reader_download.

For more assistance with Adobe Reader visit http://www.adobe.com/go/acrreader.

Windows is either a registered trademark or a trademark of Microsoft Corporation in the United States and/or other countries. Mac is a trademark
of Apple Inc., registered in the United States and other countries. Linux is the registered trademark of Linus Torvalds in the U.S. and other countries. 


\section{Please wait...}

If this message is not eventually replaced by the proper contents of the document, your PDF viewer may not be able to display this type of document.

You can upgrade to the latest version of Adobe Reader for Windows®, Mac, or Linux® by visiting http://www.adobe.com/go/reader_download.

For more assistance with Adobe Reader visit http://www.adobe.com/go/acrreader.

Windows is either a registered trademark or a trademark of Microsoft Corporation in the United States and/or other countries. Mac is a trademark
of Apple Inc., registered in the United States and other countries. Linux is the registered trademark of Linus Torvalds in the U.S. and other countries. 


\section{Please wait...}

If this message is not eventually replaced by the proper contents of the document, your PDF viewer may not be able to display this type of document.

You can upgrade to the latest version of Adobe Reader for Windows®, Mac, or Linux® by visiting http://www.adobe.com/go/reader_download.

For more assistance with Adobe Reader visit http://www.adobe.com/go/acrreader.

Windows is either a registered trademark or a trademark of Microsoft Corporation in the United States and/or other countries. Mac is a trademark
of Apple Inc., registered in the United States and other countries. Linux is the registered trademark of Linus Torvalds in the U.S. and other countries. 
Table 1. Demographic comparisons between HABS and ADNI cohorts

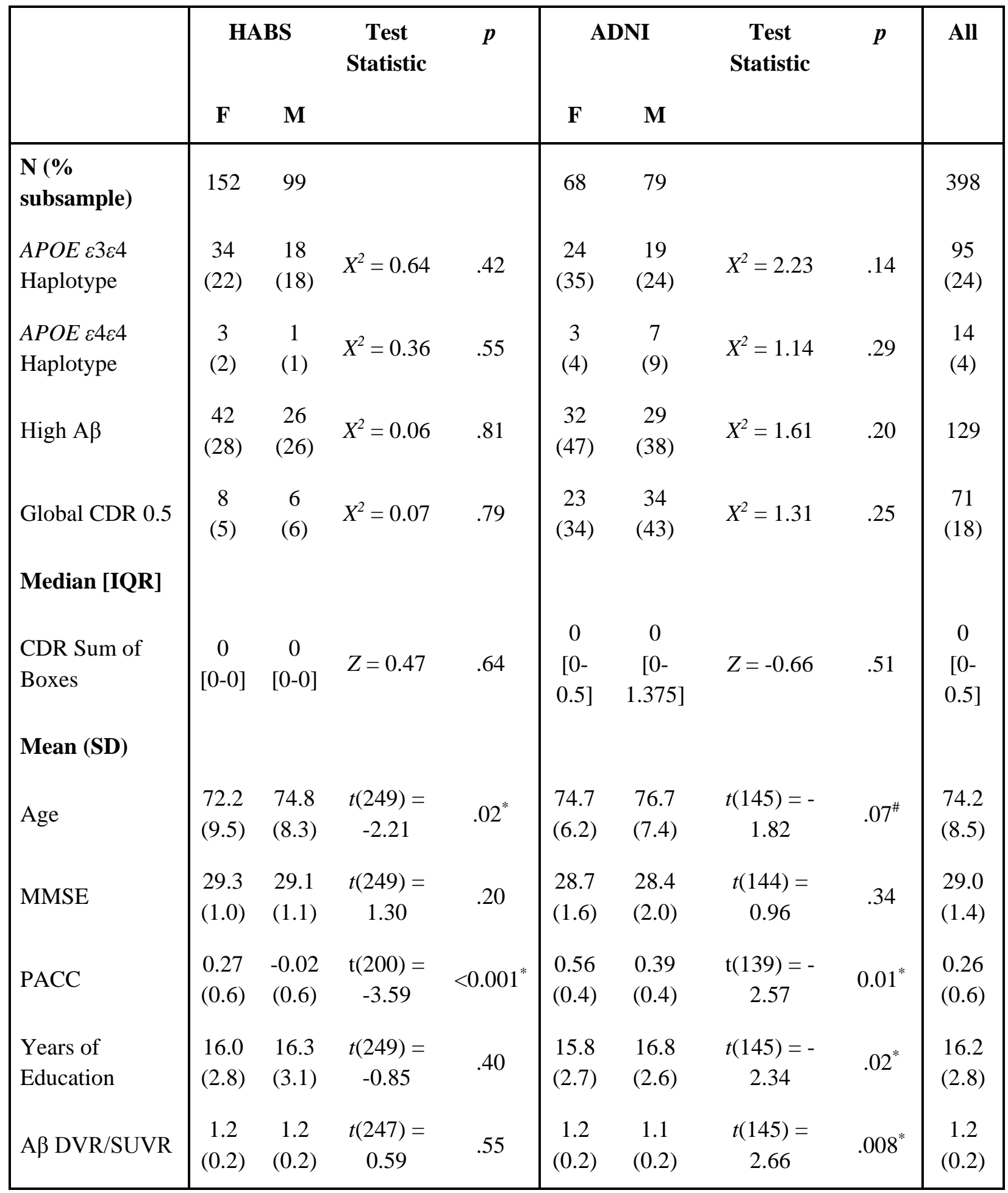

This article is protected by copyright. All rights reserved. 


\section{University Library}

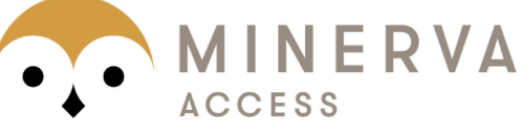

A gateway to Melbourne's research publications

Minerva Access is the Institutional Repository of The University of Melbourne

\section{Author/s:}

Buckley, RF;Scott, MR;Jacobs, HIL;Schultz, AP;Properzi, MJ;Amariglio, RE;Hohman, TJ;Mayblyum, DV;Rubinstein, ZB;Manning, L;Hanseeuw, BJ;Mormino, EC;Rentz, DM;Johnson, KA;Sperling, RA

Title:

Sex Mediates Relationships Between Regional Tau Pathology and Cognitive Decline

Date:

2020-08-31

\section{Citation:}

Buckley, R. F., Scott, M. R., Jacobs, H. I. L., Schultz, A. P., Properzi, M. J., Amariglio, R. E., Hohman, T. J., Mayblyum, D. V., Rubinstein, Z. B., Manning, L., Hanseeuw, B. J., Mormino, E. C., Rentz, D. M., Johnson, K. A. \& Sperling, R. A. (2020). Sex Mediates Relationships Between Regional Tau Pathology and Cognitive Decline. ANNALS OF NEUROLOGY, 88 (5), pp.921-932. https://doi.org/10.1002/ana.25878.

Persistent Link:

http://hdl.handle.net/11343/276206 